Quim. Nova, Vol. 34, No. 2, 226-231, 2011

\title{
AVALIAÇÃO DAS INCERTEZAS ASSOCIADAS À DETERMINAÇÃO DO PARÂMETRO DE SOLUBILIDADE DE HILDEBRAND DE PETRÓLEOS
}

\author{
Lyzette Gonçalves Moraes de Moura, Marlus Pinheiro Rolemberg e Antonio Carlos da Silva Ramos* \\ Departamento de Tecnologia Química, Universidade Federal do Maranhão, Av. dos Portugueses, s/n, Campus do Bacanga, \\ 65080-040 São Luís - MA, Brasil
}

Maria de Fátima Pereira dos Santos

Departamento de Ciências Matemáticas e Naturais, Centro Universitário Norte do Espírito Santo, Rod. BR 101 Norte, km 60 , 29932-540 São Mateus - ES, Brasil

Evaldo López Zilio

Petróleo Brasileiro S.A., Rua Almirante Barroso, 81, 30ªndar, 20030-003 Rio de Janeiro - RJ, Brasil

Recebido em 18/2/10; aceito em 20/8/10; publicado na web em 30/11/10

\begin{abstract}
EVALUATION OF THE UNCERTAINTIES ASSOCIATED TO THE DETERMINATION OF THE HILDEBRAND SOLUBILITY PARAMETER OF CRUDE OILS. Asphaltenes are fractions of crude oils that can precipitate and one of the parameters used in the prediction of the conditions in which this phenomenon occurs is the Hildebrand solubility parameter. In this work, it was evaluated the uncertainty propagation in the experimental determination of the solubility parameter of different crude oils, calculated from data of the asphaltenes precipitation by the addition of $n$-heptane, identified by optical microscopy. It was verified that the solubility parameter of an oil and the associated uncertainty are specific, being recommended that, whenever viable, it is determined parallel both, conferring higher credibility to the results.
\end{abstract}

Keywords: asphaltenes; Hildebrand solubility parameter; uncertainty propagation.

\section{INTRODUÇÃO}

Asfaltenos são frações pesadas de petróleo que durante as diversas etapas envolvidas na produção e processamento dos petróleos podem precipitar devido a alterações que ocorrem no equilíbrio de fases da mistura, decorrentes de variações de composição, temperatura e pressão. ${ }^{1,2}$

Estudos buscando uma melhor compreensão do mecanismo de precipitação estão relacionados com aspectos experimentais e teóricos e consistem no desenvolvimento de modelos termodinâmicos e coloidais que possibilitem a identificação do início da agregação dos asfaltenos no meio. ${ }^{3}$

Em alguns modelos, um dos parâmetros de entrada é o parâmetro de solubilidade de Hildebrand. ${ }^{4,5}$ No caso dos petróleos, esse parâmetro é determinado experimentalmente de forma indireta pela adição de saturados (floculantes), como o n-heptano, a petróleos ou misturas, até que a precipitação seja observada, por exemplo, por microscopia óptica. ${ }^{6}$

Nesse estudo avaliou-se a propagação de incertezas no procedimento de determinação experimental dos parâmetros de solubilidade de Hildebrand de petróleos de diferentes naturezas, buscando tanto o aperfeiçoamento do procedimento analítico, através da identificação e correção de possíveis fontes de erros, quanto uma quantificação mais realística da incerteza associada ao valor final dos parâmetros de solubilidade obtidos por essa metodologia.

\section{Revisão teórica}

Estudos de metrologia química possuem as mais diversas aplicações, que podem ser bem gerais, como o desenvolvimento e

*e-mail: ramosacs@gmail.com a utilização de programas interlaboratoriais para a qualidade dos resultados analíticos, ${ }^{7-9}$ ou mais específicas, como monitoração do teor de metais, ${ }^{10}$ caracterização de materiais candidatos a materiais de referência, ${ }^{11,12}$ medição de propriedades físicas de materiais, ${ }^{13,14}$ análises toxicológicas, ${ }^{15}$ doseamento de contaminantes em matrizes ambientais ${ }^{16}$ e validação de métodos analíticos em geral. ${ }^{15,16}$

Dependendo da natureza da medição e das informações disponíveis, estimativas de incertezas podem envolver métodos e abordagens distintos, que devem ser práticos e resultar em valores válidos. ${ }^{17-19}$ Dentre os métodos mais comuns, possivelmente o mais amplamente utilizado seja aquele apresentado no Guia para a Expressão da Incerteza de Medição, ${ }^{20}$ embora nas últimas décadas outros métodos, como a Simulação de Monte Carlo, também tenham se desenvolvido bastante. ${ }^{19,21}$ Alguns trabalhos apresentam comparações entre esses dois principais métodos e outros alternativos. ${ }^{17,18,21,22}$

Neste estudo, foram adotados os termos e métodos apresentados no Guia para a Expressão da Incerteza de Medição. ${ }^{20}$ Existem limitações e restrições neste método e na forma como é apresentado no próprio Guia, , $^{18,19,21,22}$ no entanto, alguns aspectos tornam seu emprego vantajoso, como o envolvimento de cálculos relativamente simples e a dispensa de softwares específicos. ${ }^{18}$

\section{Avaliação de incertezas}

As medições possuem imperfeições que levam a erros no resultado da medição. Tradicionalmente considera-se que um erro apresenta dois componentes, um aleatório e outro sistemático. ${ }^{23-25} \mathrm{O}$ erro aleatório pode ter origem, por exemplo, em variações temporais ou espaciais e é responsável por variações repetidas do mensurando. Este tipo de erro não pode ser compensado, mas, normalmente, pode ser minimizado aumentando-se o número de observações. O erro sis- 
temático também não pode ser eliminado, mas, por ser originário de um efeito de uma grandeza cuja influência é reconhecida no resultado da medição, pode ser quantificado e corrigido, caso seja significativo no tocante à exatidão necessária na medição.

Existem diversas fontes possíveis para a incerteza em uma medição, tais como amostragem não representativa do mensurando definido, erro pessoal na leitura/interpretação de instrumentos e resolução inadequada destes, entre outros. As fontes, quaisquer que sejam, não são necessariamente independentes e algumas podem ser fonte de outras, ademais, qualquer erro contribui para a incerteza do resultado de uma medição, mesmo que seja desconhecido e não possa ser levado em consideração na avaliação. ${ }^{16,23,24}$

O resultado de uma medição corresponde, de modo geral, apenas a uma estimativa do valor do mensurando, ou seja, da grandeza específica a ser medida, e só pode ser considerado completo quando acompanhado da declaração da incerteza dessa estimativa. ${ }^{16,23,24}$

A recomendação INC-1 (1980) do Grupo de Trabalho sobre a Declaração de Incertezas agrupa os componentes de incerteza em duas categorias, A e B, baseadas no seu método de avaliação. Ambas baseiam-se em distribuições de probabilidade e os componentes de incertezas resultantes de cada tipo são quantificados por variâncias ou desvios padrão. . $^{10,11,15,20,23,26}$

A variância pode ser interpretada como uma medida da dispersão dos resultados de medição; considerando-se $n$ observações, $\mathrm{x}_{1}, \mathrm{x}_{2}, \ldots, x_{n}$ com média aritmética $x$ (Equação 1:

$$
\bar{x}=(1 / n) \sum x_{i}
$$

A variância será dada pela soma dos desvios quadráticos das observações de sua média aritmética dividida pelo número de observações menos um, conforme expresso na Equação 2:

$$
s^{2}=[1 /(n-1)] \sum\left(x_{i}-\bar{x}\right)^{2}
$$

Esta estimativa da variância e sua raiz quadrada positiva, além do desvio padrão experimental $(s)$ caracterizam a variabilidade dos valores do mensurando observados ou, mais especificamente, sua dispersão em torno da média.

A variância estimada, $u^{2}$, que caracteriza um componente de incerteza obtido de uma avaliação do Tipo A, é calculada a partir de uma série de observações repetidas, e é a variância conhecida, $s^{2}$, estatisticamente estimada. O desvio padrão estimado, $u$, e a raiz quadrada positiva de $u^{2}$ é, portanto, $u=s$, a incerteza padrão do Tipo A. Para um componente de incerteza obtido por uma avaliação do Tipo B, a variância estimada $u^{2}$ é avaliada empregando-se o conhecimento disponível, e o desvio padrão estimado $u$ é a incerteza padrão do Tipo B. ${ }^{20,24-26}$

Para avaliações exclusivamente do Tipo A, seria necessário o desenvolvimento de uma minuciosa investigação estatística de todas as causas concebíveis de incerteza, por exemplo, empregando diversas marcas e tipos distintos de instrumentos, diferentes métodos de medição, distintas aplicações do método e diversas aproximações dos seus modelos teóricos de medição.

A avaliação da incerteza padrão do Tipo B aplica-se quando uma estimativa $x_{i}$ de uma grandeza de entrada $X_{i}$ não tenha sido obtida diretamente por meio de observações repetidas; neste caso, a variância estimada associada $u^{2}\left(x_{i}\right)$ ou a incerteza padrão $u\left(x_{i}\right)$ é avaliada por julgamento científico, baseando-se em todas as informações disponíveis sobre a possível variabilidade de $X_{i}$.

Uma descrição completa de ambos os métodos de avaliação de incerteza padrão, A e B, pode ser encontrada no Guia para a Expressão da Incerteza de Medição ${ }^{20} \mathrm{e}$ em outros trabalhos..$^{24-26} \mathrm{Os}$ componentes de incerteza presentes na determinação do parâmetro de solubilidade dos petróleos avaliados neste estudo correspondem basicamente ao Tipo B.

\section{Avaliação da incerteza padrão do Tipo B}

As avaliações do Tipo B abrangem os casos em que o mensurando $Y$ não pode ser medido diretamente, mas é determinado a partir de $N$ outras grandezas $X_{1}, X_{2}, \ldots, X_{N}$ através da relação funcional expressa na Equação 3:

$$
Y=f\left(X_{1}, X_{2}, \ldots X_{N}\right)
$$

Considere a relação funcional expressa genericamente na Equação 3. A incerteza padrão de $y$, em que $y$ é a estimativa do mensurando Y (o resultado da medição), é fornecida pela combinação adequada de incertezas padrão das estimativas de entrada $x_{1}, x_{2}, \ldots, x_{N}$. Esta incerteza padrão combinada da estimativa $y$ é representada por $u_{c}(y)$ e obtida a partir da raiz quadrada positiva da variância combinada $u_{c}^{2}(y)$, dada por:

$$
u_{c}^{2}(y)=\sum_{i=1}^{N}\left[\frac{\partial f}{\partial x_{i}}\right]^{2} u^{2}\left(x_{i}\right)
$$

em que $f$ é a função dada na Equação 3. A incerteza padrão combinada $u_{c}(y)$ é um desvio padrão estimado e caracteriza a dispersão dos valores que poderiam, razoavelmente, ser atribuídos ao mensurando $Y$. A Equação 4 expressa a Lei de Propagação da Incerteza, considerada neste estudo conforme descrita no Guia para a Expressão da Incerteza de Medição. ${ }^{20}$

As derivadas parciais $\partial f / \partial x_{i}$ equivalem a $\partial f / \partial X_{i}$ avaliadas para $X_{i=} x_{i}$. Essas derivadas são usualmente chamadas de coeficientes de sensibilidade e exprimem como a estimativa de saída $y$ varia com alterações nos valores das estimativas de entrada $x_{1}, x_{2}, \ldots$, $x_{N}$. Particularmente, a alteração em $y$, provocada por uma pequena variação $\Delta x_{i}$ na estimativa de entrada $x_{\mathrm{i}}$, é dada por $(\Delta y)_{i}=\left(\partial f / \partial x_{i}\right.$ )$\left(\Delta x_{i}\right)$. Caso tal alteração seja provocada pela incerteza padrão da estimativa $x_{\mathrm{i}}$, a variação correspondente em $y$ é $\left(\partial f / \partial x_{i}\right) u\left(x_{i}\right)$. A variância combinada $u_{c}^{2}(y)$ pode, assim, ser compreendida como a soma de termos, em que cada um deles corresponde à variância estimada associada com a estimativa de saída $y$ oriunda da variância estimada, associada com cada estimativa de entrada $x_{\mathrm{i}}$. Assim, a Equação 4 torna-se:

$$
u_{c}^{2}(y)=\sum_{i=1}^{N}\left[c_{i} u\left(x_{i}\right)\right]^{2} \equiv u^{2}\left(x_{i}\right) \sum_{i=1}^{N} u_{i}^{2}(y)
$$

em que:

$$
c_{i} \equiv \partial f / \partial x_{i}
$$

$\mathrm{e}$

$$
u_{i}(y) \equiv\left|c_{i}\right| u\left(x_{i}\right)
$$

A incerteza padrão combinada $u_{c}(y)$ é normalmente empregada para expressar a incerteza de um resultado de medição, entretanto, em determinadas aplicações comerciais, industriais e regulamentadoras, e ainda se for necessário considerar aspectos como saúde e segurança, pode ser necessário fornecer uma medida de incerteza que estabeleça um intervalo mais abrangente de valores que poderiam ser razoavelmente atribuídos ao mensurando.

Tal medida adicional de incerteza é denominada incerteza expandida $(U)$ e corresponde ao produto entre a incerteza padrão combinada $\left(u_{c}(y)\right)$ e um fator de abrangência $(k)$ : 


$$
U=k u_{c}(y)
$$

O fator de abrangência $k$ deve proporcionar um intervalo correspondente a um nível especificado de confiança $p\left(k_{p}\right)$; para tanto, é necessário que se tenha um conhecimento detalhado da distribuição de probabilidade, caracterizada pelo resultado da medição e a sua incerteza padrão combinada. No entanto, pode-se ter uma melhor aproximação substituindo este termo pelos graus de liberdade (v), que, para uma grandeza única estimada pela média aritmética de $n$ observações independentes, são geralmente iguais à Equação 9:

$$
v=n-1
$$

Quando uma grandeza de entrada é estimada a partir de outras componentes, calculam-se os graus de liberdade efetivos $\left(v_{\text {eff }}\right)$ através da fórmula de Welch-Satterthwaite, Equação 10:

$$
v_{e f f}=\frac{u_{c}^{4}(y)}{\sum_{i=1}^{N} \frac{u_{i}^{4}(y)}{v_{i}}}
$$

Se $u\left(x_{i}\right)$ for obtido de uma avaliação do Tipo A, $v_{i}$ é determinado como na Equação 9. Caso $u\left(x_{i}\right)$ venha de uma avaliação do Tipo B e puder ser tratado como exatamente conhecido, como normalmente ocorre na prática, pode-se considerar que $v_{i} \rightarrow \infty$; caso contrário, aplica-se a Equação 11:

$$
v_{i} \approx \frac{1}{2} \frac{u^{2}\left(x_{i}\right)}{\sigma^{2}\left[u\left(x_{i}\right)\right]} \approx \frac{1}{2}\left[\frac{\Delta u\left(x_{i}\right)}{u\left(x_{i}\right)}\right]^{-2}
$$

Neste caso, a avaliação do Tipo B da incerteza padrão é uma grandeza subjetiva que tem seu valor estabelecido através de julgamento científico baseado no conjunto de informações disponíveis. Assim, se, por exemplo, os conhecimentos disponíveis indicam que, para 10 observações, a avaliação de $u\left(x_{i}\right)$ é confiável cerca de $25 \%$, pode-se interpretar que o termo $\Delta u\left(x_{i}\right) / u\left(x_{i}\right)$, a incerteza relativa, é igual a 0,25 e, portanto, de acordo com a Equação 11, $v_{i}=8$.

Para obter a incerteza expandida, arredonda-se o valor $\operatorname{de}\left(v_{\text {eff }}\right)$ para o próximo número inteiro inferior, seleciona-se o nível de confiança desejado (por exemplo, 90, 95, 99\% etc.) e busca-se em uma tabela, também disponível no Guia para a Expressão da Incerteza de Medição, ${ }^{20} \mathrm{o}$ valor de $k$ correspondente. Aplicando-se a Equação 8 , obtém-se o valor da incerteza expandida.

\section{PARTE EXPERIMENTAL}

\section{Determinação experimental dos parâmetros de solubilidade dos petróleos $\left(\delta_{P}\right)$}

O parâmetro de solubilidade de Hildebrand dos petróleos $\left(\delta_{\mathrm{p}}\right)$ é o mensurando $(Y)$ e as grandezas envolvidas na sua determinação constam na relação funcional (Equação 12):

$$
\delta_{P}=f\left(\delta_{H e p}, \delta_{F}, \rho_{P}, m_{P}, V_{H e p}\right)
$$

em que $\delta_{\text {Hep }}$ é o parâmetro de solubilidade do n-heptano; $\delta_{F}$ é o parâmetro de floculação dos asfaltenos; $\rho_{P}$ é a densidade do petróleo; $m_{P}$ é a massa de petróleo e $V_{H e p}$ é o volume de n-heptano.

Na prática é muito difícil determinar diretamente os parâmetros de solubilidade dos petróleos $\left(\delta_{\mathrm{p}}\right) \mathrm{e}$, neste estudo, foram determinados indiretamente, através, basicamente, de três metodologias equivalentes.

Essas metodologias são utilizadas atualmente no CENPES (Centro de Pesquisas da Petróleo Brasileiro S.A. - CENPES/PE-
TROBRAS) para a determinação dos parâmetros de solubilidade dos petróleos extraídos e processados.

Na primeira, denominada Método I, adicionou-se n-heptano $\left(\mathrm{C}_{7} \mathrm{H}_{16}\right.$, Merck, pureza $\left.\geq 99 \%\right)$ a aproximadamente $3 \mathrm{~g}$ do petróleo em análise até que ocorresse a precipitação dos asfaltenos, confirmada por microscopia óptica, sendo este ponto denominado início de precipitação (IP).

Com esses dados, calcularam-se as frações volumétricas do petróleo e do n-heptano no ponto de início da precipitação e, posteriormente, o parâmetro de solubilidade dos petróleos $\left(\delta_{\mathrm{P}}\right)$, através da Equação 13:

$$
\delta_{P}=\frac{\delta_{F}-\delta_{H e p} \phi_{H e p}}{\phi_{P}}
$$

Os valores do parâmetro de solubilidade do n-heptano $\left(\delta_{\text {Hep }}\right)$ e o parâmetro de floculação $\left(\delta_{F}\right)$ são encontrados na literatura e foram utilizados conforme relatados. ${ }^{4,27}$ Para se determinar a fração volumétrica do petróleo $\left(\phi_{P}\right)$, converteu-se a massa do petróleo $\left(m_{P}\right)$ em volume $\left(\mathrm{V}_{\text {Hep }}\right)$ utilizando-se sua densidade, ${ }^{28}$ determinada experimentalmente no CENPES/PETROBRAS, e a Equação 13 tornou-se a 14:

$$
\delta_{P}=\frac{\delta_{F}-\delta_{H e p}\left(\frac{V_{H e p}}{\left(m_{P} / \rho_{P}\right)+V_{H e p}}\right)}{1-\left(\frac{V_{H e p}}{\left(m_{P} / \rho_{P}\right)+V_{H e p}}\right)}
$$

Quando não se verificou a precipitação de asfaltenos no petróleo pela adição de n-heptano, prosseguiu-se com a segunda metodologia, denominada Método II, em que se pesou um petróleo de referência, selecionado dentre aqueles que tiveram o parâmetro determinado através do Método I, e se adicionou o petróleo em análise, até que a precipitação ocorresse.

Neste caso, empregaram-se as frações volumétricas do petróleo de referência e do petróleo em análise, determinando-se o parâmetro de solubilidade a partir da Equação 15, análoga à Equação 13:

$$
\delta_{P}=\frac{\delta_{F}-\delta_{P R} \phi_{P R}}{\phi_{P}}
$$

em que $\delta_{R}$ e $\phi_{R}$ representam o parâmetro de solubilidade e a fração volumétrica do petróleo de referência, respectivamente. A forma da Equação 15, equivalente à 14, é a Equação 16:

$$
\delta_{P}=\frac{\delta_{F}-\delta_{P R}\left[\frac{\left(m_{P R} / \rho_{P R}\right)}{\left(m_{P} / \rho_{P}\right)+\left(m_{P R} / \rho_{P R}\right)}\right]}{1-\left[\frac{\left(m_{P R} / \rho_{P R}\right)}{\left(m_{P} / \rho_{P}\right)+\left(m_{P R} / \rho_{P R}\right)}\right]}
$$

Nos casos em que o Método II foi aplicado e se alcançou o percentual de $98 \%$ do petróleo em análise sem que se verificasse a precipitação, prosseguiu-se a análise com a terceira metodologia, Método III, que consiste em preparar uma mistura de $1: 1 \mathrm{~m} / \mathrm{m}$ de petróleo de referência e petróleo em análise e adicionar n-heptano até que a precipitação dos asfaltenos ocorra. Aqui, a variação da Equação 13 é a 17:

$$
\delta_{P}=\frac{\delta_{F}-\delta_{P R} \phi_{P R}-\delta_{H e p} \phi_{H e p}}{\phi_{P}}
$$

O Método III, na prática, difere pouco do Método I, pois, toman- 
do-se a equação para a determinação do parâmetro de solubilidade na Equação 18:

$$
\delta_{F}=\delta_{P} \phi_{P}+\delta_{P R} \phi_{P R}+\delta_{H e p} \phi_{H e p}
$$

pode-se observar que a soma $\delta_{P} \phi_{P}+\delta_{R} \phi_{R}$ representa um novo petróleo e, desse modo, as observações e conclusões decorrentes do primeiro método podem ser estendidas ao mesmo. Neste trabalho optou-se, portanto, por avaliar a propagação de erros em função da incerteza associada a cada componente empregada na obtenção do parâmetro de solubilidade apenas em relação aos Métodos I e II.

Para tanto, dentre as amostras cujos parâmetros de solubilidade foram determinados pelo Método I foram selecionados 10 petróleos (P001, P004, P008, P009, P010, P013, P014, P018, P022 e P028) e, dentre aqueles em que se aplicou o Método II, 5 petróleos (P007, P012, P039, P061 e P098). A quantidade de amostras escolhidas foi proporcional aos universos correspondentes e a seleção visou, basicamente, a diversidade de características físicas (viscosidade, densidade) e químicas (análise SARA, isto é, teores de saturados, aromáticos, resinas e asfaltenos). Na avaliação do Método I, foram realizadas 10 repetições para cada petróleo e, no Método II, 5 repetições.

\section{RESULTADOS E DISCUSSÃO}

No estudo do Método I, inicialmente foi executado o procedimento para a identificação do volume de n-heptano necessário para provocar a precipitação dos asfaltenos presentes no petróleo (IP), conforme descrito anteriormente.

Para os cálculos de incerteza padrão combinada foram necessários os valores experimentais de massa dos petróleos e os correspondentes volumes de n-heptano consumidos na precipitação, bem como as médias e desvios padrão (em relação às médias) desses termos.

A precisão e exatidão das medidas de massa e volume foram obtidas nos manuais de instrução dos equipamentos. Para as pesagens, foi utilizada uma balança analítica Shimadzu, modelo AW 220, e o valor relatado pelo fabricante refere-se à repetibilidade da medida. Nas medidas de volume, no caso do Método I, para que se obtivesse uma melhor definição do início de precipitação, foram utilizadas duas micropipetas Eppendorf de capacidades diferentes (20-200 $\mu \mathrm{L}$ e 500$5000 \mu \mathrm{L})$ e, para o cálculo das incertezas associadas ao volume total adicionado, foi necessário considerar a exatidão correspondente à pipeta e ao volume de cada adição, conforme relatados pelo fabricante.

Além desses dados, foi ainda necessário obter as derivadas parciais da Equação 14 em relação a cada componente do mensurando em questão, o parâmetro de solubilidade do petróleo $\left(\delta_{P}\right)$, a saber, $\delta_{F}, \delta_{H e p}, \rho_{P}, m_{P}, V_{H e p}$.

Obtidas estas derivadas parciais, o valor numérico correspondente a cada uma foi então determinado empregando-se os valores de cada componente, exceto em relação à massa de petróleo e ao volume de nheptano, em que foram empregadas as médias dos valores experimentais.

Com esses dados e os valores de incerteza obtidos a partir das informações fornecidas pelos fabricantes dos equipamentos de medição de massa e volume, foi possível organizar a Tabela 1, em que constam os termos necessários para a aplicação da Equação 5 na avaliação da propagação de erros para o petróleo P013.

Os desvios adotados para os parâmetros de floculação e de solubilidade do n-heptano, e também para a densidade, foram estimados a partir de valores disponíveis na literatura, ${ }^{4,27}$ respeitando-se os algarismos significativos de cada termo. Em relação ao n-heptano, considerou-se que possíveis desvios no parâmetro de solubilidade decorrentes da presença de impurezas já estariam incutidos no desvio estimado para essa grandeza.

Para estimar os graus de liberdade, considerou-se que os parâmetros de floculação e de solubilidade do n-heptano e a densidade são exatamente conhecidos e, portanto, $v_{i} \rightarrow \infty$. Quanto à massa e ao volume, por se tratarem de componentes obtidas diretamente através de observações sucessivas (Tipo A), obtiveram-se os graus de liberdade dos desvios padrão das médias das medidas pela Equação 9; já os graus de liberdade dos desvios padrão dos instrumentos de medição foram estimados através da Equação 11, considerando-se um grau (nível ou intervalo) de confiança de $25 \%$, adotada arbitrariamente ante a ausência de informações pertinentes por parte dos fabricantes.

Assim, com os valores apresentados na Tabela 1, obtém-se a incerteza padrão combinada $u\left(x_{i}\right)$ para o petróleo $\mathrm{P} 013$, por exemplo, aplicando-se a Equação 5:

$$
\begin{gathered}
u_{c P 013}^{2}(y)=\left[c_{1} u\left(\delta_{F}\right)\right]^{2}+\left[c_{2} u\left(\delta_{H e p}\right)\right]^{2}+\left[c_{3} u\left(\rho_{P}\right)\right]^{2}+\left[c_{4} u\left(m_{P}\right)\right]^{2}+\left[c_{5} u\left(V_{H e p}\right)\right]^{2} \\
u_{c P 013}^{2}(y)=0,1280^{2}+0,1516^{2}+0,0002^{2}+0,0031^{2}+0,0234^{2} \\
u_{c P 013}^{2}(y)=0,0399 M P a \Rightarrow u_{c P 013}(y)=0,2 M P a^{1 / 2}
\end{gathered}
$$

A seguir, para obter a medida adicional de incerteza, a incerteza expandida, determinaram-se os graus de liberdade efetivos para a massa e o volume, através da Equação 10:

$$
\begin{aligned}
& v_{\text {effrassa }}=\frac{u_{c}^{4}(y)}{\sum_{i=1}^{N} \frac{u_{i}^{4}(y)}{v_{i}}} \Rightarrow v_{\text {effinassa }}=\frac{0,0057^{4}}{\frac{0,0057^{4}}{9}+\frac{0,0001^{4}}{8}} \Rightarrow v_{\text {effrassa }}=9,0 \\
& v_{\text {effiolume }}=\frac{u_{c}^{4}(y)}{\sum_{i=1}^{N} \frac{u_{i}^{4}(y)}{v_{i}}} \Rightarrow v_{\text {effiolume }}=\frac{0,0675^{4}}{\frac{0,0675^{4}}{9}+\frac{0,0318^{4}}{8}} \Rightarrow v_{\text {effiolume }}=8,5
\end{aligned}
$$

\begin{tabular}{|c|c|c|c|c|c|}
\hline $\begin{array}{l}\text { Componente da incerteza } \\
\text { padrão } u\left(x_{i}\right)\end{array}$ & Fonte da incerteza & $\begin{array}{l}\text { Valor da incerteza } \\
\text { padrão } u\left(x_{i}\right)\end{array}$ & $c_{i} \equiv \partial f / \partial x i$ & $u_{i}(y) \equiv\left|c_{i}\right| u\left(x_{i}\right)$ & Graus de liberdade \\
\hline$u\left(\delta_{F}\right)$ & Desvio no parâmetro de floculação & 0,05 & 2,5605 & 0,1280 & $\infty$ \\
\hline$u\left(\delta_{\text {Hep }}\right)$ & Desvio no parâmetro de solubilidade do n-heptano & 0,1 & $-1,5605$ & 0,1560 & $\infty$ \\
\hline$u\left(\rho_{P}\right)$ & Desvio na densidade do petróleo & 0,0001 & 1,8204 & 0,0002 & $\infty$ \\
\hline$u\left(m_{P}\right)$ & Desvio na massa do petróleo & 0,0057 & $-0,5432$ & 0,0031 & \\
\hline$u\left(m_{P l}\right)$ & Desvio padrão da média das medidas & 0,0057 & & & 9 \\
\hline$u\left(m_{P 2}\right)$ & Desvio padrão do instrumento de medição & 0,0001 & & & 8 \\
\hline$u\left(V_{\text {Hep }}\right)$ & Desvio no volume de n-heptano & 0,0746 & 0,3133 & 0,0234 & \\
\hline$u\left(V_{\text {Hepl }}\right)$ & Desvio padrão da média das medidas & 0,0675 & & & 9 \\
\hline$u\left(V_{\text {Hep } 2}\right)$ & Desvio padrão do instrumento de medição & 0,0318 & & & 8 \\
\hline
\end{tabular}

Tendo-se em vista que se adotou para as outras componentes que $v_{i} \rightarrow \infty$, o valor de $\left(v_{\text {eff }}\right)$ tende a zero, logo, os graus de liberdade

Tabela 1. Sumário dos componentes da incerteza padrão na determinação do parâmetro de solubilidade de Hildebrand do petróleo P013 
efetivos do parâmetro de solubilidade do petróleo são dados por:

$$
v_{e f \delta_{p 013}}=\frac{u_{c}^{4}(y)}{\sum_{i=1}^{N} \frac{u_{i}^{4}(y)}{v_{i}}} \Rightarrow v_{e f \AA_{P 013}}=\frac{0,2^{4}}{\frac{0,0031^{4}}{9,0}+\frac{0,0234^{4}}{8,5}} \Rightarrow v_{e f \delta_{P 013}}=48705,2
$$

Com base no valor de $v_{e f f \delta P}$ e adotando-se um nível de confiança de $90 \%$, o valor tabelado de $k$ é igual a 1,645, assim:

$$
U=k u_{c}(y) \Rightarrow U_{P 013}=(1,645)\left(0,2 M P a^{1 / 2}\right) \Rightarrow U_{P 013}=0,3 M P a^{1 / 2}
$$

Consequentemente, o resultado final da medição do parâmetro de solubilidade do petróleo $\mathrm{P} 013$ pode ser declarado como:

$$
\delta_{P 013}=18,2 \pm 0,3 M P a^{1 / 2}
$$

Tabelas equivalentes à Tabela 1 foram elaboradas para os demais petróleos e os parâmetros de solubilidade com seus valores correspondentes de incerteza padrão combinada e incerteza expandida, obtidos pelos mesmos cálculos apresentados para o petróleo P013, podem ser encontrados na Tabela 2.

Tabela 2. Parâmetros de solubilidade de Hildebrand (determinados experimentalmente através do Método I), incertezas padrão combinadas e incertezas expandidas dos petróleos avaliados (P001, P004, P008, P009, P010, P013, P014, P018, P022 e P028), expressos em $M \mathrm{~Pa}^{1 / 2}$

\begin{tabular}{cccc}
\hline Petróleo & $\begin{array}{c}\text { Parâmetro } \\
\text { de solubilidade }\end{array}$ & $\begin{array}{c}\text { Incerteza } \\
\text { padrão combinada }\end{array}$ & $\begin{array}{c}\text { Incerteza } \\
\text { expandida }\end{array}$ \\
\hline P001 & 23,2 & 0,4 & 0,7 \\
P004 & 19,6 & 0,3 & 0,6 \\
P008 & 22,6 & 0,5 & 0,7 \\
P009 & 20,6 & 0,3 & 0,5 \\
P010 & 17,4 & 0,1 & 0,2 \\
P013 & 18,2 & 0,2 & 0,3 \\
P014 & 19,5 & 0,3 & 0,5 \\
P018 & 20,7 & 0,3 & 0,5 \\
P022 & 19,2 & 0,3 & 0,5 \\
P028 & 17,7 & 0,2 & 0,3 \\
\hline
\end{tabular}

Verifica-se pela Tabela 2 que a incerteza padrão combinada foi relativamente baixa, situando-se na faixa de 0,1 a 0,4 , enquanto a incerteza expandida, como seria esperado em se tratando de uma incerteza adicional, compreendeu valores maiores, entre 0,3 e 0,7.

$\mathrm{Na}$ avaliação do Método II seguiu-se basicamente o mesmo procedimento geral em relação ao tratamento estatístico dos dados. Neste caso, as derivadas parciais em função das componentes do parâmetro de solubilidade do petróleo $\left(\delta_{F}, \delta_{P R}, \rho_{P R}, \rho_{P}, m_{P R}, m_{P}\right)$ foram obtidas a partir da Equação 16.

Pelas derivadas parciais, o valor numérico correspondente a cada uma foi calculado empregando-se os valores de cada componente, somente que, no caso das massas do petróleo de referência e do petróleo em análise, foram usadas as médias dos valores experimentais. O parâmetro de solubilidade do petróleo de referência e a incerteza associada considerados nesses cálculos foram aqueles obtidos experimentalmente, conforme constam na Tabela 2.

Com esses dados e os valores de incerteza relatados pelos fabricantes do equipamento de medição de massa, organizou-se a Tabela 3, em que são apresentados os valores das componentes requeridas para a aplicação da Equação 5 na avaliação da propagação de erros para o petróleo P007.

A partir dos valores presentes na Tabela 2, realizaram-se os mesmos cálculos desenvolvidos na avaliação do Método I, já apresentados detalhadamente para o petróleo P013.

Desse modo, obteve-se que a incerteza padrão combinada do parâmetro de solubilidade do petróleo em análise, P007, calculada pela Equação 5, é igual a:

$$
u_{c P 007}(y)=0,1 M P a^{1 / 2}
$$

Os graus de liberdade efetivos da massa do petróleo em análise, P007, e da massa do petróleo de referência, P014, obtidos a partir da Equação 10 são, respectivamente:

$$
\begin{aligned}
& v_{\text {effmassaP } 007}=9,0 \\
& v_{\text {effmassaP } 014}=9,0
\end{aligned}
$$

Consequentemente, adotando-se as mesmas convenções concernentes às demais componentes $\left(v_{i} \rightarrow \infty\right)$, os graus de liberdade efetivos do parâmetro de solubilidade do petróleo P007 são dados pela Equação 10:

$$
v_{e f \delta \delta_{P 007}}=1177980,6
$$

\begin{tabular}{|c|c|c|c|c|c|}
\hline $\begin{array}{l}\text { Componente da incerteza } \\
\text { padrão } u\left(x_{i}\right)\end{array}$ & Fonte da incerteza & $\begin{array}{l}\text { Valor da incerteza } \\
\quad \text { padrão } u\left(x_{i}\right)\end{array}$ & $c_{i} \equiv \partial f / \partial x i$ & $u_{i}(y) \equiv\left|c_{i}\right| u\left(x_{i}\right)$ & Graus de liberdade \\
\hline$u\left(\delta_{F}\right)$ & Desvio no parâmetro de floculação & 0,05 & 1,1755 & 0,0588 & $\infty$ \\
\hline$u\left(\delta_{R}\right)$ & $\begin{array}{c}\text { Desvio no parâmetro de solubilidade do Petróleo de } \\
\text { Referência (PR) }\end{array}$ & 0,3 & $-0,1755$ & 0,0527 & $\infty$ \\
\hline$u\left(\rho_{P}\right)$ & Desvio na densidade do petróleo & 0,0001 & $-0,7069$ & 0,0001 & $\infty$ \\
\hline$u\left(\rho_{R}\right)$ & Desvio na densidade do Petróleo Referência & 0,0001 & 0,5666 & 0,0001 & $\infty$ \\
\hline$u\left(m_{P}\right)$ & Desvio na massa do petróleo & 0,0315 & 0,1195 & 0,0038 & \\
\hline$u\left(m_{P I}\right)$ & Desvio padrão da média das medidas & 0,0315 & & & 9 \\
\hline$u\left(m_{P 2}\right)$ & Desvio padrão do instrumento de medição & 0,00028 & & & 8 \\
\hline$u\left(m_{R}\right)$ & Desvio na massa do petróleo de referência (P014) & 0,0052 & $-0,5457$ & 0,0031 & \\
\hline$u\left(m_{R I}\right)$ & Desvio padrão da média das medidas & 0,0052 & & & 9 \\
\hline$u\left(m_{R 2}\right)$ & Desvio padrão do instrumento de medição & 0,0001 & & & 8 \\
\hline
\end{tabular}

Também neste caso foi adotado um nível de confiança de $90 \%$ e o valor tabelado de $k$, sendo igual a 1,645 para o grau de liberdade encontrado, fornece, através da Equação 8, uma incerteza expandida do parâmetro de solubilidade do petróleo em análise, P007, igual a:

Tabela 3. Sumário dos componentes da incerteza padrão na determinação do parâmetro de solubilidade de Hildebrand do petróleo P007 


$$
U_{P 007}=0,1 \mathrm{MPa}^{1 / 2}
$$

Portanto, o resultado final da medição do parâmetro de solubilidade do petróleo P007 pode ser declarado como:

$$
\delta_{P 007}=15,9 \pm 0,1 M P a^{1 / 2}
$$

Tabelas equivalentes à Tabela 3 foram elaboradas para os demais petróleos (P012, P039, P061 e P098) cujos parâmetros de solubilidade foram determinados através do Método II e as respectivas incertezas padrão combinadas e incertezas expandidas obtidas para todos, inclusive para o P007, podem ser encontradas na Tabela 4 , juntamente aos parâmetros de solubilidade correspondentes a cada petróleo avaliado nesta etapa.

Tabela 4. Parâmetros de solubilidade de Hildebrand (determinados experimentalmente através do Método II), incertezas padrão combinadas e incertezas expandidas dos petróleos avaliados neste estudo (P007, P012, P039, P061 e P098), expressos em $\mathrm{MPa}^{1 / 2}$

\begin{tabular}{cccc}
\hline Petróleo & $\begin{array}{c}\text { Parâmetro } \\
\text { de solubilidade }\end{array}$ & $\begin{array}{c}\text { Incerteza padrão } \\
\text { combinada }\end{array}$ & $\begin{array}{c}\text { Incerteza } \\
\text { expandida }\end{array}$ \\
\hline P007 & 15,9 & 0,1 & 0,1 \\
P012 & 16,0 & 0,1 & 0,1 \\
P039 & 16,1 & 0,1 & 0,1 \\
P061 & 16,3 & 0,1 & 0,1 \\
P098 & 15,9 & 0,1 & 0,2 \\
\hline
\end{tabular}

Também aqui foi adotado um nível de confiança de 90\%. Para os petróleos $\mathrm{P} 007, \mathrm{P} 012$, $\mathrm{P} 039$ e $\mathrm{P} 061$, o valor tabelado de $k$, para os graus de liberdade calculados, é igual a 1,645; para o petróleo P098, obteve-se $v_{\text {eff́p } 098}=70,3$ e o valor tabelado de $k$, neste caso, é igual a 1,670 .

Observa-se na Tabela 4 que tanto a incerteza padrão combinada quanto a incerteza expandida foram baixas, situando-se na faixa de 0,1 a 0,2. Comparando-se com os valores apresentados para os petróleos cujos parâmetros de solubilidade foram determinados pelo Método I, verifica-se que a incerteza é bem inferior neste caso, provavelmente devido às menores incertezas associadas ao instrumento de medição, pois, em relação aos petróleos do Método I, torna-se necessário considerar, além do desvio da balança, os das pipetas empregadas, relativamente maiores, como se pode observar comparando-se os mesmos nas Tabelas 1 e 3, enquanto que em relação aos petróleos do Método II, devem-se considerar apenas os desvios associados à balança.

\section{CONCLUSÕES}

Verificou-se que tanto o parâmetro de solubilidade, quanto a incerteza associada à sua determinação são aspectos característicos de cada petróleo. Por conveniência, poder-se-ia adotar a incerteza máxima encontrada em cada caso (Método I e Método II) como sendo, genericamente, uma incerteza padrão associada à determinação experimental deste parâmetro, independentemente da natureza do petróleo.

Observou-se, ainda, que mesmo os petróleos analisados por um dado método apresentam características bastante diferentes em relação à composição e densidade, o que é um indício de que talvez não seja possível agrupá-los relacionando suas propriedades físicoquímicas com o método utilizado na determinação do parâmetro de solubilidade correspondente.
Estudos posteriores, todavia, poderão envolver uma caracterização mais detalhada dos petróleos e considerar a possibilidade de se estabelecer o controle do processo analítico para cada um os métodos (I, II e III) em função de possíveis características comuns aos petróleos analisados por cada metodologia, definindo a incerteza final associada aos respectivos processos analíticos.

Recomenda-se, no entanto, que, sempre que experimentalmente viável, se realize o procedimento executado no desenvolvimento deste trabalho, determinando-se paralelamente o parâmetro de solubilidade e a incerteza padrão combinada, ou a incerteza expandida, para cada petróleo, individualmente, conferindo, deste modo, maior credibilidade aos resultados obtidos.

\section{REFERÊNCIAS}

1. Asomaning, S.; Pet. Sci. Technol. 2003, 21, 581.

2. Vicente, L.; Soto, C.; Pacheco-Sánchez, H.; Hernández-Trujillo, J.; Martinez-Magadán, J. M.; Fluid Phase Equilib. 2006, 239, 100.

3. Ramos, A. C. S.; Tese de Doutorado, Universidade Estadual de Campinas, Brasil, 2001.

4. Wiehe, A. I.; Kennedy, R. J.; Energy Fuels 2000, 14, 56.

5. Wong, G. K.; Yen, T. F.; J. Pet. Sci. Eng. 2000, $28,55$.

6. Moura, L. G. M.; Santos, M. P. F.; Zilio, E. L.; Rolemberg, M. P.; Ramos, A. C. S.; J. Pet. Sci. Eng. 2010, 74, 77.

7. Chui, Q. S. H.; Antonoff, H. B.; Olivieri, J. C.; Quim. Nova 2002, 25, 657

8. Chui, Q. S. H.; Bispo, J. M. A.; Iamashita, C. O.; Quim. Nova 2004, 27, 993.

9. Chui, Q. S. H.; Barros, C. B.; Silva, T. D.; Quim. Nova 2009, 32, 2209.

10. Chui, Q. S. H.; Zucchini, R. R.; Lichtig, J.; Quim. Nova 2001, 24, 374.

11. Gardner, J. L.; J. Res. Natl. Inst. Stand. Technol. 2004, 109, 305.

12. Chui, Q. S. H.; Iamashita, C. O.; Bispo, J. M. A.; Quim. Nova 2005, 28, 497.

13. Barbato, G.; Germak, A.; Low, S.; Proceedings XVII IMEKO World Congress, Dubrovnik, Croácia, 2003.

14. Rabin, Y.; Cryobiology 2003, 46, 109.

15. Lima, I. M. H.; Frota, M. N.; Quim. Nova 2007, 30, 1820.

16. Damas, E. Y. C.; Medina, M. O. C.; Clemente, A. C. N.; Díaz, M. A. D.; Bravo, L. G.; Ramada, R. M.; Porto, R. M. O.; Quim. Nova 2009, 32, 855 .

17. Oliveira, E. C.; Aguiar, P. F.; Quim. Nova 2009, 32, 1655.

18. Jornada, D. H.; Caten, C. T.; Resumos do V Congresso Latino Americano de Metrologia (METROSUL), Curitiba, Brasil, 2007.

19. Oliveira, E. C.; Aguiar, P. F.; Quim. Nova 2009, 32, 1571.

20. BIPM; IEC; IFCC; ISSO; IUPAC; OIML; Guia para a expressão da incerteza de medição, $3^{\mathrm{a}}$ ed., ABNT/INMETRO: Rio de Janeiro, 2003.

21. Donatelli, G. D.; Konrath, A. C.; Rev. Ciencia Tecnol. 2005, 13, 5.

22. Moscati, G.; Mezzalira, L. G.; Santos, F. D.; Resumos do ENQUALAB 2004 - Encontro para Qualidade de Laboratórios, São Paulo, Brasil, 2004.

23. Blank, A. B.; J. Anal. Chem. 2005, 60, 1173.

24. Lira, F. A.; Metrologia na Indústria, $7^{\mathrm{a}}$ ed., Érica: São Paulo, 2009.

25. Figliola, R. S.; Beasley, D. E.; Theory and Design for Mechanical Measurements, $4^{\text {th }}$ ed., Wiley: New York, 2006.

26. http://www.nist.gov/, acessada em Fevereiro e Dezembro 2010.

27. Hansen, C. M.; Hansen Solubility Parameters: a User's Handbook, $2^{\text {nd }}$ ed., CRC Press LLC: Boca Raton, 2000.

28. ISO 12185:1996, Crude petroleum and petroleum products Determination of density - Oscillating U-tube method, International Organization for Standardization, Geneva. 